\title{
Microcin-mediated Interactions Between Klebsiella pneumoniae and Escherichia coli Strains
}

\author{
By VÍCTOR DE LORENZO, * JOSÉ L. MARTÍNEZ AND CARLOS ASENSIO† \\ Instituto de Enzimologia y Patología Molecular (CSIC), Facultad de Medicina de la Universidad \\ Autónoma de Madrid, Madrid 34, Spain
}

(Received 5 July 1983; revised 29 September 1983)

\begin{abstract}
Amensal indirect interactions between a Klebsiella pneumoniae microcin-producing strain and several Escherichia coli strains, all of intestinal origin, were studied. Mixed batch cultures of both microcin-producing and microcin-sensitive strains showed that microcin production and excretion into the medium allowed the producer strain to prevail over sensitive strains, even when initial competition conditions were highly unfavourable for the producer. Mixed cultures also showed the production of a microcin-antagonist by the same microcin-producing strain when the nutrients in the medium had been depleted. The antagonist apparently promoted the viability of sensitive cells already damaged by microcin. These results have likely ecological implications.
\end{abstract}

\section{INTRODUCTION}

Quantitative features of microbial indirect relations mediated by inhibitory agents have been widely analysed (de Freitas \& Fredrickson, 1978), but few experimental studies are available concerning antagonism between antibiotic-producing strains and other micro-organisms which share common habitats (Helling et al., 1981; Adams et al., 1979).

The human intestinal tract lodges a rather well-defined community of micro-organisms that show dynamic successions both in the species composition and in the relative abundance of each resident, under either normal or pathological conditions. These successions are frequently very fast and specific for some invaders, which are able in some way to displace other very closely related species by means of poorly understood mechanisms (Savage, 1977). Bacteriocin production has been suggested to be involved in amensal relations which would account for phenomena observed in this ecosystem such as implantation ability, strain displacements, colonization-resistance and many others. Nevertheless, no evidence supporting these hypotheses has so far been provided (Konisky, 1978).

The inhibitors called microcins are other candidates that may explain bacterial displacements in the intestinal microbial ecosystem. Microcins are low molecular weight antibiotics produced by Enterobacteriaceae isolated from human faeces (Asensio et al., 1976). Over 10\% of the Escherichia coli and Klebsiella strains isolated from this source are microcinogenic. In accordance with their characteristics (low molecular weight compared with bacteriocins, production and activity in poor media, resistance to extremes of $\mathrm{pH}$ and certain proteases), it has been proposed that microcins are involved in the maintenance of homeostasis in the intestinal ecosystem (Baquero \& Asensio, 1979; Aguilar et al., 1982a).

This paper introduces the study of indirect amensal interactions between a Klebsiella pneumoniae microcin-producing strain and microcin-sensitive $E$. coli strains, all of intestinal origin. Mixed batch cultures of producer and sensitive strains offer indications that interaction is mediated both by the inhibitory action of excreted microcin and by the release of a microcinantagonist by the same microcin-producer strain when the nutrients in the culture are depleted.

$\dagger$ Deceased. 


\section{METHODS}

Bacterial strains. Micro-organisms used are described in Table 1. Klebsiella pneumoniae RYC492 is a clinical isolate of faecal origin found in a screening of microcin-producing strains (Asensio et al., 1976), performed at the Microbiology Department at the Centro Especial Ramon y Cajal in Madrid by A. Fernandez and F. Baquero. In both liquid and solid media, this strain produces a diffusible peptide (mol. wt approximately 5000), a bacterial inhibitor that we have termed microcin 492 or M492. This microcin is especially active on enterobacteria, but does not affect the producer strain. Klebsiella pneumoniae RYC492 harbours two plasmids of 2.6 and 37 MDal, and there are some preliminary results relating M492 production to the smaller plasmid (V. de Lorenzo, unpublished).

Non-producing mutants derived from $K$. pneumoniae RYC492 were obtained by treatment with $N$-methyl- $N^{\prime}$ nitro- $N$-nitrosoguanidine as described by Adelberg et al. (1965) and were termed A301, A302, R301, R302, A303 and A305. Polyauxotrophic E. coli AB1157 was kindly provided by Dr F. Moreno. Escherichia coli RK6 is a microcin-resistant mutant derived from $E$. coli BM21. Escherichia coli B was used as a strain hyper-sensitive to microcin 492. Its derivative, $E$. coli IERl, is a spontaneous rifamycin-resistant strain.

Media. Minimal medium M9 (Clowes \& Hayes, 1968), supplemented with $0 \cdot 2 \%(\mathrm{w} / \mathrm{v})$ glucose and $0 \cdot 001 \%(\mathrm{w} / \mathrm{v})$ thiamin chloride was used. Rich media were BHI (Brain Heart Infusion, Difco) and LB (Luria Broth); solid and semisolid (soft agar) media were prepared by adding, respectively, $1.5 \%$ and $0.7 \%(\mathrm{w} / \mathrm{v}$ ) agar (Difco) to the corresponding liquid medium. When soft agar medium was used to obtain crude preparations of microcin, it was prepared by adding $0.5 \%(\mathrm{w} / \mathrm{v})$ of type $A$ agarose (Pronadisa) to $\mathrm{M} 9$ medium. Selective media for strains resistant to kanamycin, nalidixic acid and rifamycin were prepared by adding to the described media filter-sterilized solutions of these antibiotics to reach final concentrations of 13,40 and $150 \mu \mathrm{g} \mathrm{ml}^{-1}$, respectively.

Microcin production in anaerobiosis. The production of the antibiotic under anaerobic conditions was tested as previously described (Asensio et al., 1976). BBL Gas-Pak jars (Becton-Dickinson) were used to achieve an anaerobic environment. Escherichia coli BM21 was used as the sensitive strain; E. coli RK6 (resistant to microcin) and $K$. pneumoniae R302 (non-producing mutant) were introduced in the assays as controls of specificity of sensitivity and production, respectively.

Mixed cultures of producer and sensitive strains. Bacterial growth was followed by measuring the $\mathrm{OD}_{660}$ with a Bausch \& Lomb colorimeter. Erlenmeyer flasks $(150 \mathrm{ml})$, fitted with side-arms and containing $10 \mathrm{ml}$ of the appropriate media, were inoculated separately with single colonies of the required strains previously grown on minimal medium plates and incubated at $37^{\circ} \mathrm{C}$ with shaking to reach an $\mathrm{OD}_{660}$ of 0.1 (about $4 \times 10^{7} \mathrm{cells}^{-1}$ ). Suitable fractions of each culture, producer and sensitive cells, were then mixed in sterile $25 \mathrm{ml}$ flasks to achieve the desired initial cellular ratios of the various strains in a final volume of $10 \mathrm{ml}$. Mixed cultures were again incubated at $37^{\circ} \mathrm{C}$ with shaking and the titres of each strain were monitored by plating samples of the culture on agar with LB or BHI (total titre), LB plus nalidixic acid (E. coli K 12 titre), LB plus kanamycin (K. pneumoniae titre) or LB plus rifamycin ( $E$. coli IER1 titre).

When the polyauxotroph $E$. coli AB1157 was used as the sensitive strain, the inoculum was grown on M9 medium supplemented with each of the amino acids required for growth, at $40 \mu \mathrm{g} \mathrm{ml}^{-1}$. When this inoculum reached an $\mathrm{OD}_{660}$ of $0 \cdot 1$, cells were centrifuged for $30 \mathrm{~min}$ at $2000 \mathrm{~g}$, resuspended in the same volume of fresh medium without amino acids and mixed with microcin-producer strain as described above.

Kinetics of action 'in vivo' for microcin 492: survival curves. Standard suspensions of sensitive cells were prepared as follows. An overnight culture of $E$. coli BM21 was diluted 20-fold in fresh M9 medium, and incubated with

\section{Table 1. Bacterial strains}

Species

Escherichia coli $\mathrm{K} 12$

Escherichia coli B

Klebsiella pneumoniae

\section{Strain}

BM21

AB 1157

RK6

NCIB9484

IER 1

RYC492

A301

A302

R301

R302

A303

A 305
Relevant phenotype*

Prototrophic, $\mathrm{Nal}^{\mathrm{R}}$

Thr, Leu, His, Pro, Arg, Nal ${ }^{R}$

Prototrophic, $\mathrm{Nal}^{\mathrm{R}}$, $\mathrm{Mcc}^{\mathrm{R}}$

Prototrophic

Prototrophic, Rif ${ }^{R}$

$\mathrm{Km}^{\mathrm{R}}, \mathrm{Mcc}^{+}, \mathrm{Mcc}^{\mathrm{imm}+}$

$\mathrm{Km}^{\mathrm{R}}, \mathrm{Mcc}^{-}, \mathrm{Mcc}^{\mathrm{imm}+}$

$\mathrm{Km}^{\mathrm{R}}, \mathrm{Mcc}^{-}, \mathrm{Mcc}^{\mathrm{imm}+}$

$\mathrm{Km}^{\mathrm{R}}, \mathrm{Mcc}^{-}, \mathrm{Mcc}^{\mathrm{imm}+}$

$\mathrm{Km}^{\mathrm{R}}, \mathrm{Mcc}^{-}, \mathrm{Mcc}^{\mathrm{imm}+}$

$\mathrm{Km}^{\mathrm{R}}, \mathrm{Mcc}^{-}, \mathrm{Mcc}^{\mathrm{imm}+}$

$\mathrm{Km}^{\mathrm{R}}, \mathrm{Mcc}^{-}, \mathrm{Mcc}^{\mathrm{imm}+}$
Origin

F. Baquero

F. Moreno

This work

CECT 101

This work

This work

This work

This work

This work

This work

This work

This work

* $\mathrm{Nal}^{\mathrm{R}}$, Resistance to nalidixic acid; $\mathrm{Rif}^{\mathrm{R}}$, resistance to rifamycin; $\mathrm{Km}^{\mathrm{R}}$, resistance to kanamycin; $\mathbf{M c c}^{\mathrm{R}}$, resistance to microcin 492 ; $\mathrm{Mcc}^{+}$, microcin production; $\mathrm{Mcc}^{\mathrm{imm}+}$, immunity to microcin. 
shaking at $37^{\circ} \mathrm{C}$ until the mid-exponential phase $\left(\mathrm{OD}_{660}=0 \cdot 3\right)$ was reached. Then $8 \mathrm{ml}$ of the culture was centrifuged at $2000 \mathrm{~g}$ for $30 \mathrm{~min}$, and the pellet was resuspended in $2 \mathrm{ml} \mathrm{M} 9$ medium without glucose. This standard suspension (approximately $10^{\circ}$ c.f.u. $\mathrm{ml}^{-1}$ ) was stored at $5^{\circ} \mathrm{C}$ until use. Such cells apparently maintained their metabolic situation at exponential conditions for at least $10 \mathrm{~h}$.

Survival curves were constructed in the following way. Samples $(4 \mathrm{ml})$ from a culture of microcin-producing strain in M9 medium were taken at suitable times during growth and mixed in $25 \mathrm{ml}$ sterile Erlenmeyer flasks with $100 \mu \mathrm{l}$ of the standard sensitive cell suspensions giving approximately $2.5 \times 10^{7} \mathrm{c.f} . \mathrm{u} . \mathrm{ml}^{-1}$. Flasks were subsequently incubated at $37^{\circ} \mathrm{C}$ with shaking and the changes in the viability of sensitive cells in the mixed culture were followed by plating samples at desired times on LB-nalidixic acid agar. When survival curves were made with supernatants, the culture of microcin-producing strain (taken at desired times), was centrifuged at $5^{\circ} \mathrm{C}$ for $30 \mathrm{~min}$ at $2000 \mathrm{~g}$ and the supernatant was either supplemented with nalidixic acid $\left(40 \mu \mathrm{g} \mathrm{ml}^{-1}\right)$ to avoid growth of remaining $K$. pneumoniae cells, or filter-sterilized using polycarbonate membranes with $0.4 \mu \mathrm{m}$ pore size (Bio Rad). A $4 \mathrm{ml}$ portion of this supernatant was added to $100 \mu \mathrm{l}$ standard sensitive cell suspension and the viability was followed as before.

Protease sensitivity was tested at a final protein concentration of $100 \mu \mathrm{g} \mathrm{ml}^{-1}$. All proteases were purchased from Sigma. Trypsin was the type treated with diphenyl carbamyl chloride (DPCC) to avoid chymotrypsin contamination.

Microcin preparations. Crude cell-free preparations of microcin were obtained by the following procedure. M9 melted soft agar medium was inoculated at $42^{\circ} \mathrm{C}$ with $4 \times 10^{3} \mathrm{c}$.f.u. K. pneumoniae $\mathrm{RYC} 492 \mathrm{ml}^{-1}$, and poured into sterile Petri dishes. The plates were incubated overnight at $37^{\circ} \mathrm{C}$ and the agar was triturated and centrifuged at $20000 \mathrm{~g}$ for $30 \mathrm{~min}$. The supernatant was filter-sterilized as before; it showed an antibiotic activity of about 2000 arbitrary antibiotic units $\mathrm{ml}^{-1}$ against $E$. coli BM21, as measured by the critical dilution method (Asensio et al., 1976). Further details concerning microcin purification and characterization will be published elsewhere (V. de Lorenzo, unpublished).

Microcin antagonist assay. A crude microcin preparation $(0.25 \mathrm{ml})$ was spread on the surface of a Petri dish containing $25 \mathrm{ml} \mathrm{M9}$ solid medium. The plate was then spray-seeded with a suspension of $E$. coli BM21 sensitive strain. Strains being tested for antagonist production were inoculated into the agar by means of toothpicks. Petri dishes were incubated at $37^{\circ} \mathrm{C}$ for $40 \mathrm{~h}$ under aerobic or anaerobic conditions. Antagonist was considered present when the sensitive strain formed a circle of growth around the spot of the tested strain.

\section{RESULTS}

\section{Mixed cultures $E$. coli/K. pneumoniae}

Figure 1(a) shows the changes in the viability of the cells in a mixed culture of $E$. coli BM21 and K. pneumoniae RYC492 in minimal medium, with a starting ratio of microcin-producing to sensitive cells of $1: 1$. The microcinogenic strain titre rose regularly, whereas that of the sensitive strain fell in $3 \mathrm{~h}$ to reach its lowest level, three orders of magnitude below the initial conditions. From that point on, a rapid recovery of the viability of the sensitive cells was observed, which became stable in the following $3 \mathrm{~h}$. When $E$. coli IER $1\left(\mathrm{Rif}^{\mathrm{R}}\right)$ was used as the sensitive strain, similar patterns were observed (data not shown). In all cases, no relevant changes of $\mathrm{pH}$ were detected so far in the culture medium during the development of mixed cultures (pH 6.9 to 6.6).

Figure $1(c)$ shows the same experiment performed in rich medium, BHI. Under these conditions, there was an inhibition in the growth of the sensitive strain without apparent killing of the cells. This could be due to a decreased sensitivity of the cells to microcin in rich media and/or to a lower concentration of the antibiotic. Examples of these two possibilities have been described for other microcins (Aguilar et al., 1982b).

Non-producing mutants, such as $K$. pneumoniae $\mathrm{R} 302$, were unable to produce any inhibitory effects on sensitive cells in either minimal or rich medium (Figs $1 b$ and $d$, respectively). On the other hand, the patterns showed by the mixed cultures between microcin-producing $K$. pneumoniae RYC492 and microcin-resistant $E$. coli RK6 were fully similar to those described in Figs $1(b)$ and $(d)$, in the sense that the growth of $E$. coli-resistant cells was not affected by the presence of microcin-producing strains. The same results were obtained when other $\mathrm{Mcc}^{-}$or $\mathrm{Mcc}^{\mathrm{R}}$ mutants were used in mixed cultures (data not shown).

Mixed cultures were set up in minimal medium starting with the producing/sensitive cells ratio favourable to the latter $(1: 10$ or $1: 100)$. The plots in Fig. 2 show that the microcinproducing strain prevailed over the sensitive strain in the mixed culture even under such unfavourable initial conditions. Nevertheless, when the ratio producer/sensitive strain was yet 


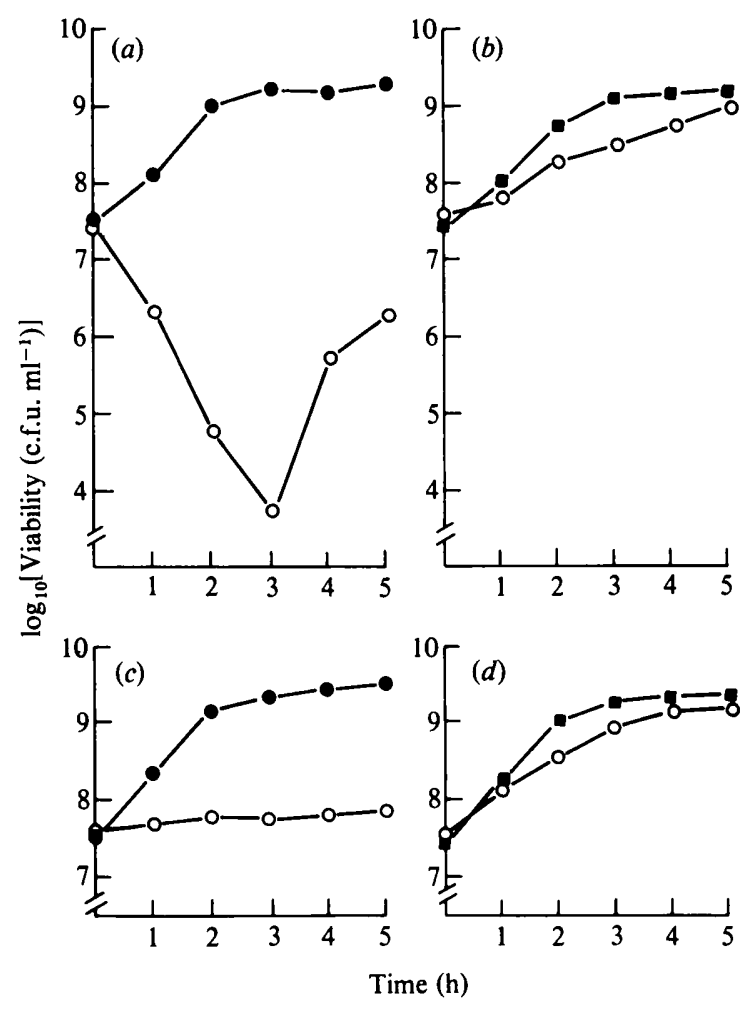

Fig. 1. Mixed culture of $K$. pneumoniae RYC492 and E. coli BM21 in different media. Strains were grown separately in either minimal or rich medium to reach an $\mathrm{OD}_{660}$ of $0 \cdot 1$. Equal volumes of each strain were then mixed. Strain titres were obtained by plating samples of the mixed culture on LBnalidixic acid ( $E$. coli BM21 titre) and LB-kanamycin (K. pneumoniae RYC492 or R302 titre). Klebsiella pneumoniae $\mathrm{R} 302$ is a non-producing mutant (see Methods). (a) Mixed culture of $K$. pneumoniae RYC492 (O) and $E$. coli BM21 (O) in minimal medium. (b) Mixed culture of $K$. pneumoniae R302 ( $\square$ ) and $E$. coli BM21 $(O)$ in minimal medium. (c) Mixed culture of $K$. pneumoniae RYC492(O) and $E$. coli BM21 (O) in rich medium BHI. (d) Mixed culture of $K$. pneumoniae R302 ( $\square$ ) and E. coli BM21 (O) in rich medium BHI.

lower, i.e. 1:1000 (Fig. 2d), neither mortality of the sensitive strain nor prevalence of producer strain was detected, probably due to the low microcin concentration in the medium when the glucose had already been depleted by the growth of the sensitive strain.

The recovery of viability of the sensitive strain was observed in mixed cultures at times longer than $3 \mathrm{~h}$ (Fig. 1 a). This recovery could not be attributed to the overgrowth of microcin-resistant cells, as indicated by analysis of the viable sensitive cells isolated from the mixed culture at late stages of the producer-strain growth. When the polyauxotrophic $E$. coli $\mathrm{AB} 1157$ strain was used as sensitive strain in conditions preventing its growth, viability recovery was still observed (Fig. 3). This indicated that recovery could not be attributed to cellular growth processes, but to some kind of rescue mechanism. This rescue seemed to be mediated by a microcin-antagonist which appeared in the medium when glucose was depleted. Experimental evidence supporting this hypothesis is shown below. This last experiment also showed that cell growth is not required for microcin action because killing took place whether or not the cells were growing.

\section{Survival curves}

Killing kinetics of sensitive cells were studied by constructing survival curves at shorter times. These curves were obtained by mixing subcultures of the microcin-producing strain, taken at different stages of growth, with a constant number of sensitive cells (Fig. 4). The highest 

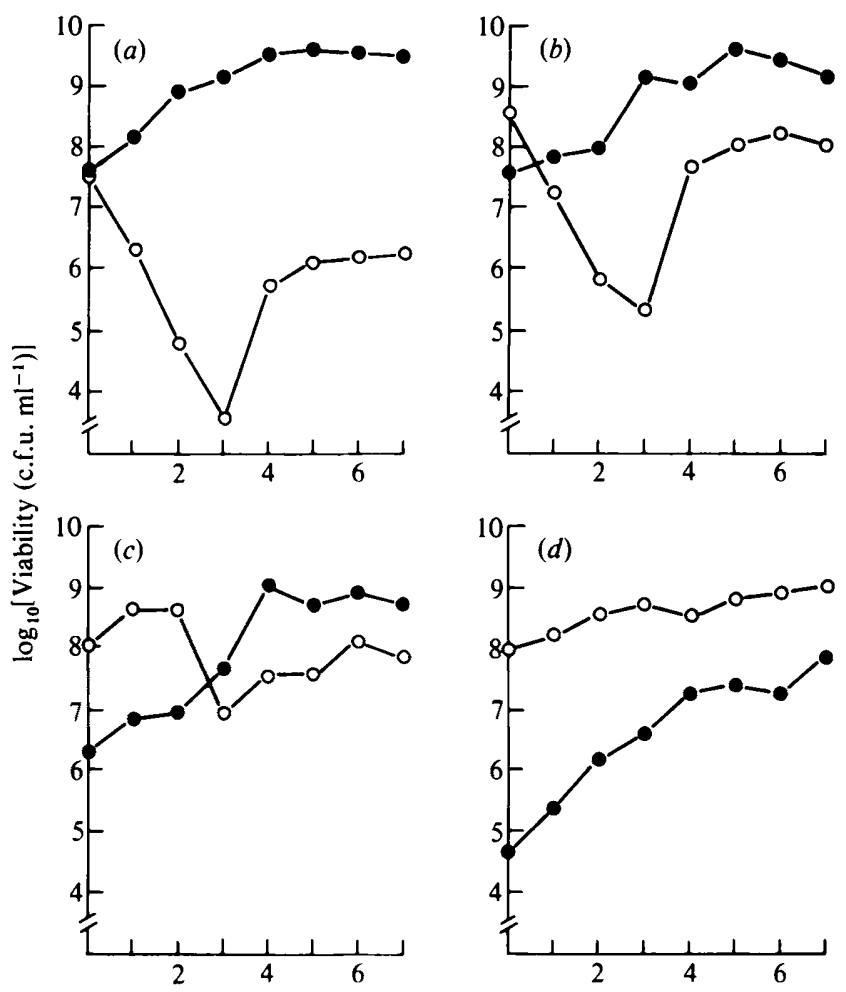

Time (h)

Fig. 2. Mixed cultures of $K$. pneumoniae RYC492 and $E$. coli BM21 with different initial ratios. Cells were grown separately in minimal medium to reach an $\mathrm{OD}_{660}$ of $0 \cdot 1$. At that point, appropriate volumes of each strain were mixed to get an initial ratio producer/sensitive cells of $1: 1(a), 1: 10(b), 1: 100(c)$ and $1: 1000(d)$. K. pneumoniae RYC492; O, E. coli BM21.

mortality was observed with subcultures of the microcin-producing strain obtained in its exponential phase of growth, whilst no mortality was observed with subcultures from the stationary phase. These results are consistent with those shown in Figs 1 and 2, in which it can be seen that the rescue began only when the mixed culture was about to reach the stationary phase. On the other hand (Fig. 4), a close parallelism can be observed in the mortality patterns of the microcin-sensitive strain in the presence or absence of producer cells. When the changes in the viability of the cells in this kind of mixed culture were followed for longer periods (Fig. 5), it was observed that recovery of viability was possible only when microcin-producing cells were present. When producer cells were removed from the culture by centrifugation, or their growth was inhibited at early stages of the exponential phase (when we presumed that there was no significant production of the microcin inhibitor), recovery was not detected or, in any case, was much slower than when microcin-producing cells were present (Fig. 5). This kind of experiment showed that the microcin-antagonist is a diffusible agent and that it is released into the medium by the same producer cells at late stages of growth.

Microcin activity was quickly inactivated by pronase and chymotrypsin (Fig. $6 a$ ), but not by trypsin (Fig. $6 b$ ). This last protease apparently exerted an activation effect, which is especially noteworthy in the situation plotted in Fig. 6(c). In this case, the subculture of the microcinproducing strain was obtained at the stationary phase of growth in which microcin activity can no longer be detected in the absence of the protease. This activation effect could be attributed to an inactivation of the microcin-antagonist by trypsin rather than to a direct action on the microcin itself, since purified microcin is insensitive to trypsin at the same concentration (V. de Lorenzo, unpublished). These experiments suggest that the antagonist could contain peptide linkages. 


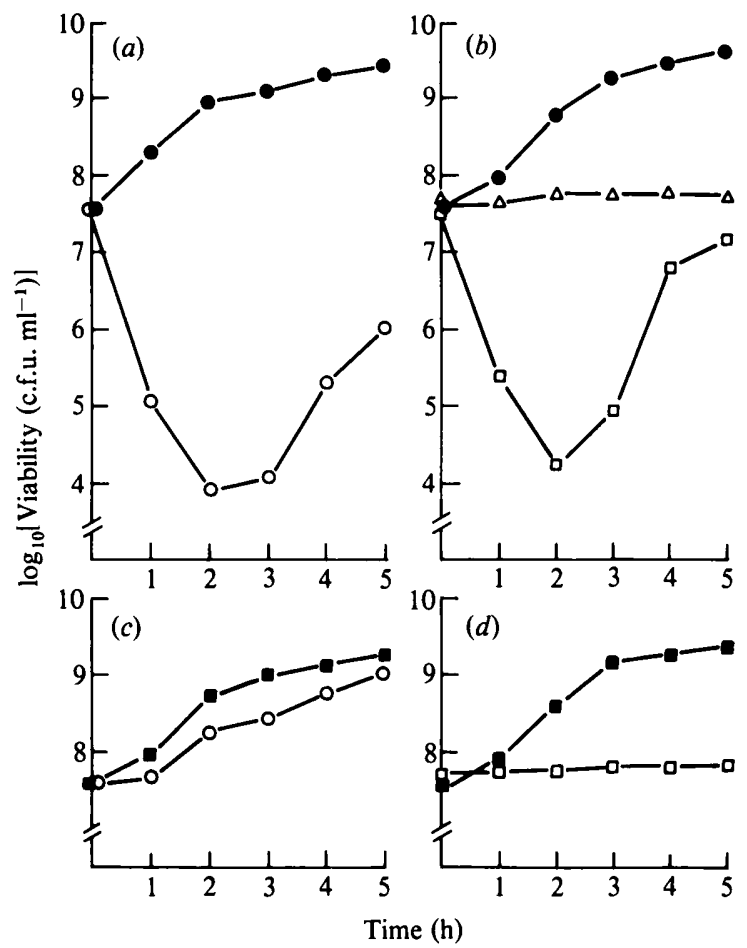

Fig. 3. Mixed cultures of $K$. pneumoniae RYC492 and polyauxotrophic $E$. coli AB1157. Mixed cultures were prepared as described in Methods. Escherichia coli AB1157 was grown in M9 medium supplemented with the amino acids required for growth up to an $\mathrm{OD}_{660}$ of $0 \cdot 1$. Cells were then centrifuged, resuspended in the same volume of minimal medium without amino acids and mixed with the appropriate strain of $K$. pneumoniae. All the following mixed cultures were prepared in minimal medium. (a) Control mixed culture of $K$. pneumoniae RYC492 (O) and E. coli BM21 (O). (b) Mixed culture of $K$. pneumoniae RYC492 (O) and E. coli AB1157 ( $\square$ ). As control, the viability of $E$. coli $\mathrm{AB} 1157(\triangle)$ was followed in minimal medium without amino acids. (c) Mixed culture of non-producing mutant $K$. pneumoniae R302 ( $\square$ ) and E. coli BM21 (O). (d) Mixed culture of $K$. pneumoniae R302 ( $\square$ ) and E. coli $\mathrm{AB} 1157(\square)$.
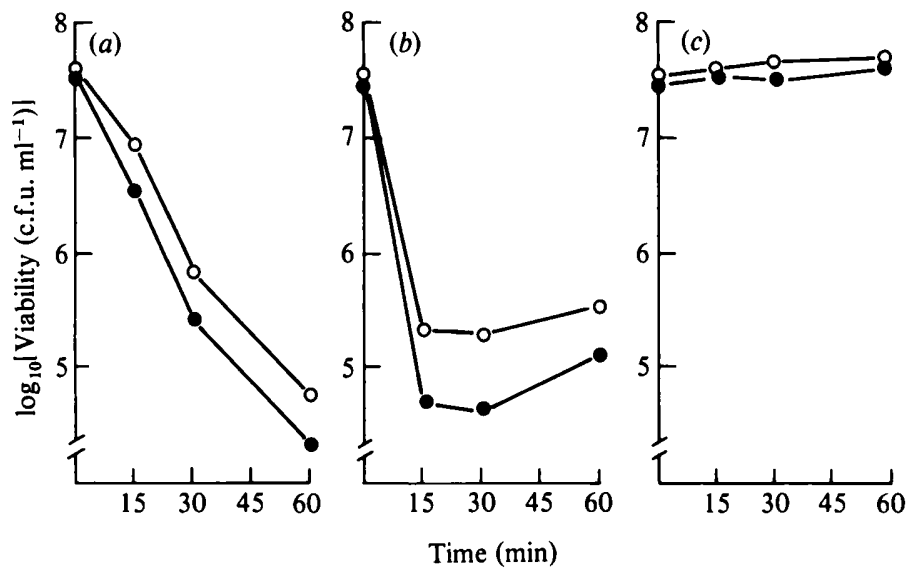

Fig. 4. Survival curves of $E$. coli BM21 in cultures of $K$. pneumoniae RYC492 and their supernatants obtained at different stages of growth. Subcultures from a growing $K$. pneumoniae RYC492 culture in minimal medium were taken at different stages of growth. Standard suspensions of $E$. coli BM21 were mixed each time with the subculture and with the corresponding subculture supernatant. The viability of $E$. coli BM21 either in the subcultures with producer cells $(O)$ or in the supernatants $(O)$ was followed by plating samples in LB-nalidixic acid. Subcultures were taken at an $\mathrm{OD}_{660}$ for the producer strain of: $0.3(a), 0.8(b)$ and $1.0(c)$. 


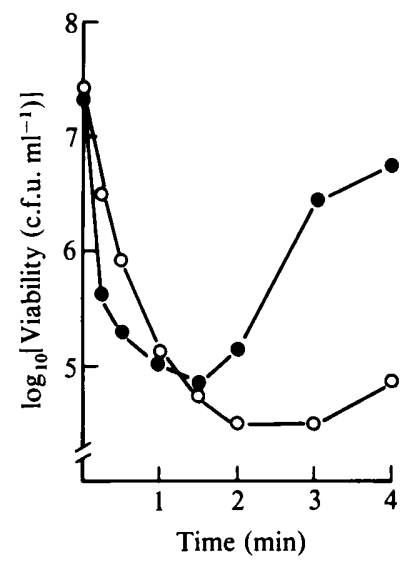

Fig. 5. Viability of $E$. coli BM21 in a culture of $K$. pneumoniae RYC492 and in its supernatant. Klebsiella pneumoniae $\mathrm{RYC} 492$ was grown to reach an $\mathrm{OD}_{660}$ of 0.37 . At that point, one half of the culture was centrifuged to remove producer cells. Standard suspensions of $E$. coli BM21 were added to the supernatant and to the culture with producer cells. Viability of $E$. coli BM2l was followed as described in Methods. Titre of $E$. coli BM21 in the culture with producer cells; $O$, the same, in the supernatant.
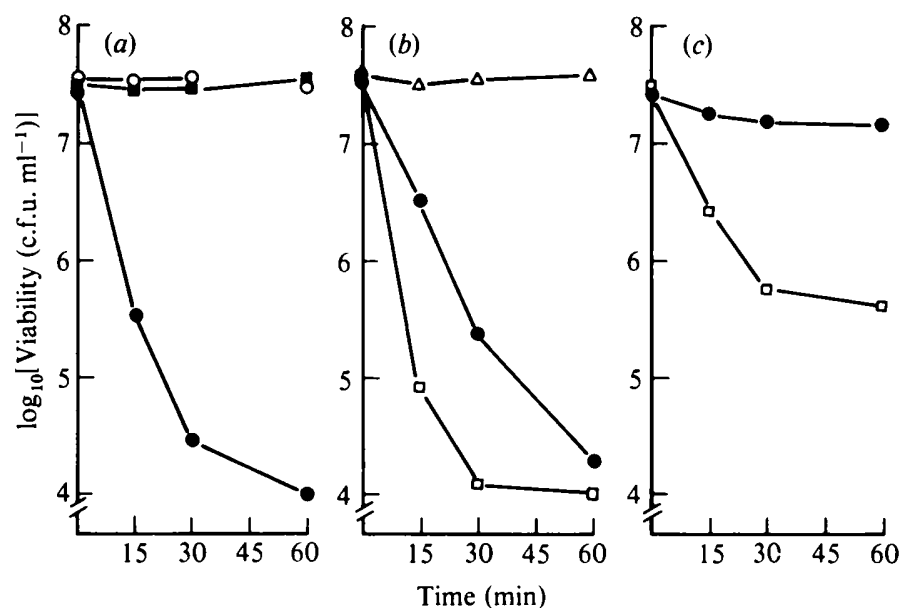

Fig. 6. Survival curves of $E$. coli BM21 in cultures of $K$. pneumoniae RYC492 treated with proteases. Standard suspensions of $E$. coli BM21 were mixed with subcultures of $K$. pneumoniae RYC492 in minimal medium in different conditions: (a) The subculture of $K$. pneumoniae RYC492 was taken at an

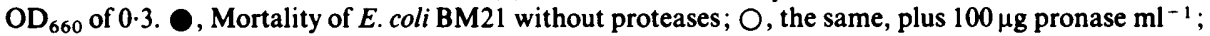
口, the same, plus $100 \mu \mathrm{g}$ chymotrypsin $\mathrm{ml}^{-1}$. (b) The subculture was taken as before at an $\mathrm{OD}_{660}$ of $0 \cdot 3$.

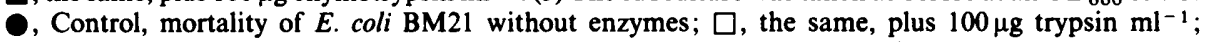
$\triangle$, viability of $E$. coli $\mathrm{BM} 21$ in minimal medium plus $100 \mu \mathrm{g}$ trypsin $\mathrm{ml}^{-1}$, without producer cells. (c) The subculture was taken at the stationary phase at an $\mathrm{OD}_{660}$ of 1.0 . O, Mortality of E. coli BM21 without protease; $\square$, the same, plus $100 \mu \mathrm{g}$ trypsin $\mathrm{ml}^{-1}$.

\section{Plate assay of the microcin-antagonist}

Further evidence for the production of a microcin-antagonist by the microcin producer strain was obtained as shown in Fig. 7. Mutants from $K$. pneumoniae RYC492 which failed to produce microcin were still able to release into the medium a diffusible agent which allowed the growth of sensitive cells around their spots on plates supplemented with microcin. Similar results were obtained when this assay was performed under anaerobic conditions. 


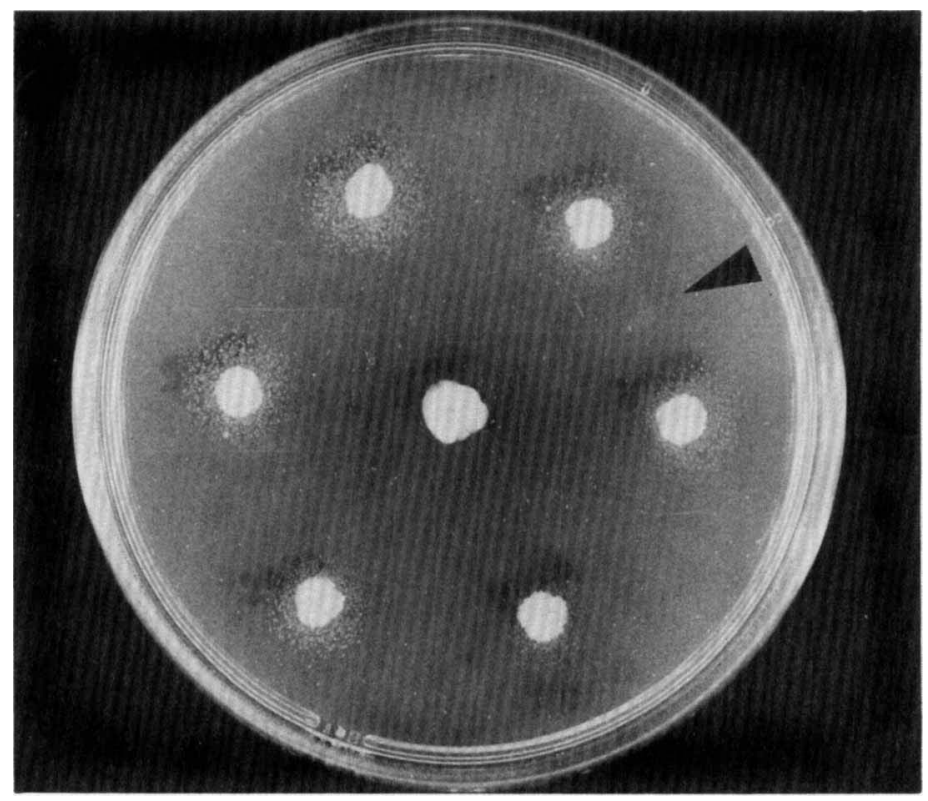

Fig. 7. Production of microcin-antagonist by $K$. pneumoniae mutants which fail to produce M492. A Petri dish containing M9 minimal agar medium was supplemented with approximately 100 antibiotic units per ml of M492 obtained as described in Methods. Non-producing mutants of $K$. pneumoniae were then inoculated into the agar by means of a toothpick (anticlockwise from arrow: K. pneumoniae A301, A302, R301, R302, A303 and A305). The plate was spray-seeded with a suspension of the sensitive strain E. coli BM21 and incubated for $40 \mathrm{~h}$. The central spot is a control of $K$. pneumoniae RYC492.

\section{Microcin activity in anaerobic conditions}

Plate assays showed that $K$. pneumoniae RYC492 produced microcin under anaerobic conditions. An inhibition zone was observed around the colony of the producer strain similar to that observed in the same experiment performed in aerobic conditions. The non-producing mutant $K$. pneumoniae R302 did not inhibit the sensitive strain $E$. coli BM21 under anaerobic conditions. Similarly, there was no inhibition zone when the resistant strain $E$. coli RK6 was tested for sensitivity to microcin. These controls ruled out the possibility that inhibition was due to causes other than microcin production and sensitivity to microcin in anaerobiosis.

\section{DISCUSSION}

The production of antibacterial substances by a large number of Klebsiella strains was occasionally described in the early studies on bacteriocins (Durlakowa et al., 1964). In most cases, these inhibitors have been indiscriminately named bacteriocins from Klebsiella or klebocins and have been classified (Maresz-Babczszyn et al., 1967) according to heterogeneous criteria such as resistance/sensitivity to high temperature, proteases or chloroform, and inducibility by UV light. Strain typing by means of these klebocins has been successfully used in clinical work for epidemiological studies (Buffenmyer et al., 1976). However, until now none of these Klebsiella bacteriocins has been purified or characterized and some of these substances formerly described as bacteriocins could actually be considered as microcins, since in current screening tests these two kinds of substances are virtually indistinguishable if differentiation assays (Asensio et al., 1976) are not performed. On the other hand, studies on indirect interactions between micro-organisms mediated by antibacterial substances have been rather limited (Adams et al., 1979) and no report is available in the literature concerning the interactions mediated by inhibitors produced by Klebsiella. The interest in this last point arises 
mainly from the wide spread of Klebsiella nosocomial infections which might be attributable in some cases to imbalances in the bacterial ecosystems where this kind of micro-organism usually resides (Eickhoff, 1979).

When the microcin producer strain $K$. pneumoniae RYC492 was grown in liquid minimal medium mixed with an $E$. coli $\mathrm{K} 12$ strain, there was a net prevalence of the producer over the sensitive strain even when initial conditions were highly favourable to the latter. We ruled out the possibility that the prevalence of $K$. pneumoniae RYC492 was due to non-specific factors ( $\mathrm{pH}$ changes, nutrient depletion, non-specific inhibitions) and consider the cause to be the release of a microcin into the medium, since non-producing mutants isolated from the RYC492 strain were unable to affect the growth of sensitive strains, and microcin-resistant cells (RK6 strain) grew unaffected by the presence of $K$. pneumoniae RYC492. When the producer strain reached the stationary phase, there was a recovery of viability in the sensitive strains. This was apparently due to microcin inhibition by a diffusible antagonist produced by the same $K$. pneumoniae RYC492 strain and not to the proliferation of resistance or any other kind of growth or cellular process in the sensitive cells.

Excretion into the medium by Enterobacteriaceae (and other micro-organisms) of biological inhibitors, together with antagonists of these inhibitors, has been described in some cases (Foulds \& Shemin, 1969; Davie \& Brock, 1966; Aguilar et al., 1982a) although no special significance has been ascribed to this fact. In our case, both antibacterial agent and antagonist are apparently synthesized and released into the medium by the same strain at different stages of its growth cycle. The microcin-antagonist exerts its action only when nutrients in the medium are depleted, but during the exponential growth of the producer strain the microcin activity appears to predominate. The antagonist apparently acts in cells already damaged by microcin, since this factor appears not to inactivate the microcin molecule itself.

A point which remains to be studied is the possible involvement of the microcin-antagonist in the immunity mechanisms of the microcin-producing strain against it own product. In this case, recovery of viability by the sensitive strain would be an effect derived from the self-protecting mechanism of producer cells.

The results of the experiments described in our in vitro amensalism/competition system suggest a mechanism of interaction between antibiotic-producer and sensitive strains which may have some significance in natural bacterial ecosystems. In this case, interaction would be mediated by the balance between the microcin and the microcin-antagonist, both released to the medium by the same producer strain in a ratio determined by the availability of nutrients in the system.

Both microcin production and sensitivity to the antibiotic were also observed under anaerobic conditions, as well as excretion of a microcin-antagonist by the same producer strain. This activity of the microcin in anaerobiosis is consistent with its proposed role in natural microbial interactions, although factors other than microcin production (namely generation times, activity of proteases and fermentation products) should be taken into account to relate our results to the phenomena observed in the intestinal ecosystem.

Further evidence to support the hypotheses above mentioned would require purification and characterization of the microcin-antagonist molecule as well as ecological work in more complex systems. These two topics are currently under study in this laboratory.

The authors are indebted to Drs F. Baquero, A. Aguilar and C. F. Heredia for critical reading of the manuscript; to Drs F. Moreno and J. L. San Millan for essential methodological suggestions and to N. Hopwood for correcting the manuscript. Technical assistance of Amalia Montes and Manuel Molina is also acknowledged. This work was supported by a grant from Fondo de Investigaciones Sanitarias de la Seguridad Social (1981).

\section{REFERENCES}

AdAMS, J., KinNey, T., Thompson, S., RubiN, L. \& Hellin, R. B. (1979). Frequency-dependent selection for plasmid containing cells of Escherichia coli. Genetics 91, 627-637.

Adelberg, E. A., Mandel, M. \& Ching Chen, C.
(1965). Optimal conditions for mutagenesis by $N$ methyl- $N^{\prime}$-nitro- $N$-nitrosoguanidine in Escherichia coli K12. Biochemical and Biophysical Research Communications 18, 788-795.

Aguilar, A., Perez-Diaz, J. C. \& Asensio, C. 
$(1982 a)$. Mechanisms involved in the increased sensitivity of Escherichia coli to microcin $15 \mathrm{~m}$ at $42^{\circ} \mathrm{C}$. Current Microbiology 7, 83-86.

Aguilar, A., Perez-Diaz, J. C., Baquero, F. \& AsENSIO, C. (1982b). Microcin 15m from Escherichia coli: mechanism of antibiotic action. Antimicrobial Agents and Chemotherapy 21, 381-386.

Asensio, C., Perez-Diaz, C., Martinez, M. C. \& BAQUERO, F. (1976). A new family of low molecular weight antibiotics from Enterobacteria. Biochemical and Biophysical Research Communications 69, 7-14.

Baquero, F. \& Asensio, C. (1979). Microcins as ecological effectors in human intestinal flora: preliminary findings. In New Criteria for Antimicrobial Therapy, pp. 90-94. Edited by D. van der Waaij \& J. Verhoef. Amsterdam: Excerpta Medica.

BuffeNMYer, C. L., RYCHECK, R. \& YeE, R. B. (1976). Bacteriocin (Klebocin) sensitivity typing of Klebsiella. Journal of Clinical Microbiology 4, 239-244.

Clowes, R. C. \& HAYES, W. (1968). Experiments in Molecular Genetics. New York: Wiley.

DAVIE, J. M. \& Brock, T. D. (1966). Effect of teichoic acid on resistance to the membrane lytic agent of Streptococcus zymogenes. Journal of Bacteriology 92 , 1623-1631.

Durlakowa, I., Maresz-Babczyszyn, J., PrzondoHessex, A., lusar, Z. \& Mroz-Kurpiela, E. (1964). The phenomenon of bacteriocinogeny in bacilli of the genus Klebsiella: characteristics of
Klebsiella bacteriocins. Archivum immunologiae et therapiae experimentalis 12, 308-318.

EICKHOFF, T. C. (1979). Nosocomial respiratory tract infections: the gastrointestinal tract as a reservoir. In New Criteria for Antimicrobial Therapy, pp. 5-11. Edited by D. van der Waaij \& J. Verhoef. Amsterdam: Excerpta Medica.

FouldS, J. D. \& SHEmIN, D. (1969). Concomitant synthesis of a bacteriocin and a bacteriocin inactivator from Serratia marcescens. Journal of Bacteriology 99, 661-666.

de Freitas, M. J. \& Fredrickson, A. G. (1978). Inhibition as a factor in the maintenance of the diversity of microbial ecosystems. Journal of General Microbiology 106, 307-320.

Helling, R. B., Kinney, T. \& Adams, J. (1981). The maintenance of plasmid containing organisms in populations of Escherichia coli. Journal of General Microbiology 123, 129-141.

KonISKY, J. (1978). The bacteriocins. In The Bacteria, vol. 6, pp. 71-136. Edited by L. N. Ornston \& J. R. Sokatch. New York: Academic Press.

Maresz-Babczyszyn, J., Durlakowa, I., LachoWICZ, Z. \& HAMON, Y. (1967). Characteristics of bacteriocins produced by Klebsiella bacilli. Archivum immunologiae et therapiae experimentalis 15, 530 539.

Savage, D. C. (1977). Microbial ecology of the gastrointestinal tract. Annual Review of Microbiology 31, 107-133. 